\title{
Mindfulness and Spirituality: Therapeutic Perspectives
}

\author{
Asimina Lazaridou ${ }^{\text {a }}$ \& Panagiotis Pentaris ${ }^{\text {b,c }}$
}

a Department of Anesthesiology, Perioperative and Pain Medicine, Brigham and Women's

Hospital, Harvard Medical School, Boston, MA 02155, USA

${ }^{\mathrm{b}}$ Faiths \& Civil Society Unit (FCSU) \& Department of Social, Therapeutic and Community

Studies, Goldsmiths, University of London, 23 St. James Road, London SE14 6NW, UK

${ }^{\text {c }}$ Department of Social Work \& Integrated Care, Bucks New University, UK

Corresponding author: Panagiotis Pentaris, MSc, p.pentaris@gold.ac.uk, Faiths \& Civil Society Unit (FCSU) \& Department of Social, Therapeutic and Community Studies Goldsmiths, University of London, 23 St. James Road, London SE14 6NW, UK, +44(0)2077172541. 


\begin{abstract}
Social aspects that relate to beliefs and spirituality are subjects of the soul and mind, material to be studied out of the materialistic world. They transcend the body and nest in the experiences of the soul. Nonetheless, taught by mindfulness, a practice that stems from eastern spirituality the religious and/or the spiritual are experiences lived through the body. We conducted a pilot crosssectional study to test that hypothesis that mindfulness correlate with spiritual beliefs. The results provide insight on the potential impact of mindfulness interventions in patients that value spirituality and metacognitive beliefs in the psychotherapeutic process. These preliminary findings provide a potential insight into the possible mechanisms underlying the application mindfulness in psychotherapy.
\end{abstract}

Keywords: spirituality, mindfulness, embodiment, religion, body awareness 


\section{Introduction}

It is surprising and unforeseen that more and more research is undertaken around spirituality. Either as a concept or a trait, spirituality, when addressed independently from religion and religiousness, has no particular history of inclusion in scientific discourse, hence surprising. Due to its lack of scientific status, little attention has been given to it, however this is in large changing nowadays (Egan et al., 2011; George, Larson, Koenig \& McCullough, 2000).

Spirituality and religion have been linked and studied as similar concepts by a lot of scientists, albeit often times have been separated and approached independently (Canda \& Furman, 2010). Spirituality has been distinguished from religiousness and religious practice where individual’s purpose in life focuses on personal growth and inner understanding. Nonetheless, this article will not be focusing on the definitional or conceptual disparities between religion and spirituality, even though references will be made throughout. To the contrary, this paper focuses on an exploration of mindfulness and spirituality from a therapeutic perspective.

There is evidence suggesting that mindfulness can be effective for spiritual development (Walsh, 1992; Wallace \& Shapiro, 2006). Mindfulness is defined as paying attention on purpose, in the present moment and, non-judgmentally, to the unfolding of experience moment to moment (KabatZinn et al., 1990). Even though perfectly accurate, this definition unfortunately doesn't fully illuminate how mindfulness can be linked to spirituality and personal growth and what are the mechanisms that lead mindfulness practitioners to a balanced life.

With its roots in Buddhist meditation, a secular practice of mindfulness has entered the American mainstream in recent years, in part through the work of Jon Kabat-Zinn and his MindfulnessBased Stress Reduction (MBSR) program, which he launched at the University of Massachusetts 
Medical School in 1979. There is a high degree of overlap in the early stages of mindfulness practice, both in a secular or a Buddhist context.

With that in mind, a non-religious person may as well experience the present moment in full awareness of their spirit and mind. Further with this essay, we are integrating lived religion (Day, 2010) and spiritual practices in the discussion to demonstrate how spirituality in a broader sense links to mindfulness. This will set the context in which this pilot study takes place, and the data are interpreted.

\section{Spirituality}

Weaver et al. (2006) suggest that despite the arguments around the linkages between religion and spirituality, there is common understanding that both concepts are different, but still interconnected. The latter has been argued by a number of scholars (Koenig, 2008). Koenig (2008) suggests that religion has disentwined from spirituality, and therefore those two can be addressed separately. Extant research shows that an entrenched attempt is undertaken in order to study spirituality outside of the realm of religiousness and religious practice. Two examples that illustrate the above are the following. Berry (2005), who states that the subjective nature of spirituality should not be the reason why it cannot be studied. Secondly, Swinton and Pattinson (2010, p.226) state that "the vagueness of the concept...is its strength and value". Both examples support the treatment of spirituality as an independent area of research.

Tanyi (2002) suggests the subjectified nature of the experience of the spiritual, which goes to speak beyond the contemporary meanings and descriptions of spirituality, but meet the indigenous explanations of it; for example, the Maori people of New Zealand (Rochford, 2004) or the indigenous people of the State of Hawai'i (Pentaris, 2011). 
Tanyi's argument is in line with how spirituality has been placed in clinical practice and therapeutic approaches. "Today, more and more individuals are seeking greater meaning and fulfillment in their lives, believing that spirituality is vital for their growth and essential for helping them deal with life’s problems” (Sperry, 2001, p.1). Sperry (ibid.) goes on to explore key questions that rise in the journey of introducing the spiritual in clinical practice, and notably psychotherapy. One of the aims of her account is to understand whether it is appropriate and professionally sanctioned to incorporate spirituality in psychotherapy. Indeed, Harris et al. (2015) explored the interplay of faith, including spirituality, in moral injury regarding military mental health. In their work, the authors conclude that spirituality is an integral part of the individual's life, and thus shall be considered in psychotherapy. Also, the authors conclude that spirituality is an essential tool when developing and exercising interpersonal skills.

Research supports that each person has a spiritual life, and that every patient in clinical practice thinks of their spiritual care as important as their psychosocial and physical (Mueller et al., 2001). Similarly, Sperry (2001, p.3) agrees that clients in psychotherapy expect “clinicians to incorporate the spiritual dimension”. According to Steere (1997), individuals do not merely seek emotional and physical healing, but spiritual as well. It is worth noting, however, that often notions of spirituality overlap with religion, and also mindfulness. The latter appears to carry characteristics not so different from spirituality. Mindfulness, however, is not necessarily linked to a religious creed, but it definitely employs a spiritual attitude towards the well-being of the individual, which is experienced through the body (McGuire, 2003).

In the next section we are reviewing literature that investigates mindfulness as a spiritual and/or religious practice. This will provide an established understanding of the relationship between mindfulness and spirituality to share therapeutic perspectives. 


\section{Mindfulness as a spiritual practice}

Among others definitions, meditation is defined as an ancient spiritual practice and the central component of mindfulness, which aims to still the mind by eliminating negative thoughts, inducing a state of relaxation. Although there are many different schools and types of meditation - connected to formal philosophical systems and/or religious practices- the various forms and practices can be seen as variations of concrete operationalization of meditation and its philosophy. Given a simpler definition meditation is being concentrated on a fixed point. Therefore, when compared to other states and processes of the mind, these differences may be disregarded as inessential in comparison with the common nucleus that characterizes meditation in general (Baerentsen et al., 2010). In terms of the larger topic of meditation, the most common other type involves purposeful attention on a particular object, image, phrase, or word. This form of meditation is designed to lead to a subjective experience of absorption with the object of focus dissolution of the differentiation of self and other. There is another distinction in which meditation is guided by following along with a leader who verbally directs the practitioner, either in person or on tape. Others merely practice meditation on their own volition. From a spiritual point of view, mindfulness meditation is considered to be a therapeutic tool within a larger program aimed at eliminating all suffering in life, and, thus, whether psychologically healthy or not, every human being is hypothesized to gain from practicing mindfulness meditation.

However, Kabat-Zinn’s mindfulness meditation program (1990) is not affiliated with any religious order, even though it originates from a spiritual tradition, Vipassana, which in turn emanated from oriental philosophical teachings. Therefore, Mindfulness has been used in a lot of different scientific programs and therapies such as MBSR (Mindfulness Based Stress Reduction), 
DBT (Dialectical Behavioral Therapy), MBCT (Mindfulness Based Cognitive Therapy) and ACT (Acceptance and Commitment Therapy).

\section{Locating mindfulness in spirituality and religion}

The engagement of religion, spirituality and mindfulness in the same conversation hinders numerous challenges and controversies. The main challenge is twofold. First and foremost, mindfulness as a trait or a concept is naturally located in Asian religious denominations and practices, and is hardly identified as the same within different contexts. However, Ricoeur's (1976) position in religion and poetics enhances the possibilities for relocating spirituality within mindfulness and viceversa. Hettema (2011), using some of Ricoeur's philosophy, aims to explore in-depth some elements of spiritual hermeneutics. Spirituality is suggested to be experienced in a subjective and individual manner, and thus how the individual becomes mindful through their spiritual stance in life becomes an intimate encounter. Similar to Day's (2010) thesis of performative religion, mindfulness is demonstrated in a performative fashion, albeit the contextual differences across nations, cultures, traditions, and religious denominations and practices. Otherwise referred to as the lived religion, its intersection with other social aspects of the individual's identity (or social identities of the individual) amounts to different experiences, nonetheless this does not relate to lack of mindfulness and/or mindful experiences through the body. 


\section{Pilot Study}

Our main aim in this study was to explore the relationship between spiritual beliefs and mindfulness. Therefore, we conducted a pilot study and collected preliminary data assessing the association of spiritual expressions and the five factors of mindfulness.

\section{Methods}

We collected data through convenience sampling from 100 participants through social media. We recruited 100 (response rate: 96\%) through social media and asked them to complete questions on spirituality and mindfulness. Out of 95 participants included in the final analysis, 68\% were in a relationship (including married and engaged). The age range of the participants was between 21 and 58 years old $(M=31.61, S D=9.24)$. Out of 100 participants, 65.3\% were female, 55.8\% single, 70\% were European residents, 21\% USA residents, 57.9\% native English speakers, 45.3\% had a Masters degree and 53.7\% were between 26-34 years old (Table 1). All statistical analysis was performed using SPSS v.18.0 (IBM Corp., Armonk, NY, USA). We ran Pearson correlations to explore the relationship between mindfulness and spiritual expressions.

\section{Measures}

The Five Factor Mindfulness Questionnaire (FFMQ). The five factors of mindfulness are measured by a 39-item scale called Five Factor Mindfulness Questionnaire (Baer et al., 2006). These five factors are as follows: a) Observing (attending to or noticing internal and external stimuli, such as feelings, cognitions, sights, sounds, and smells); b) Describing (noticing or mental word-tagging of these stimuli); c) Acting with awareness and consciousness (attending to one's current actions in contrast to behaving automatically or unconsciously); d) Non-judging the inner 
experience (abstaining from assessment of one's feelings, cognitions, and emotions); (e.) Nonreactivity to the inner experience (allowing thoughts and feelings to come and go, without being engaged in them). Responses to the items fall on a 5-point Likert-type scale (1= never or very rarely true, $5=$ very often or always true). The five subscales exhibit an adequate to good internal consistency. To use this scale for an Iranian population, similar steps were taken. The test-retest was administered on 30 participants with a 2-week interval and showed a consistency of 0.74 between the two administrations and proved significant at the level of 0.001 . The Cronbach's alpha coefficient of the test stood at 0.89 as well.

Expressions of Spirituality Inventory (MacDonald, 2000). The inventory consists of 32 items in five subscales measuring the five dimensions of spirituality identified by MacDonald (2000): Cognitive Orientation To- wards Spirituality; Experiential/Phenomenological Dimension; Existential Well-being, Paranormal Beliefs, and Religiousness. Cognitive Orientation Towards Spirituality pertains to the expressions of spirituality which refer to cognitive thoughts, beliefs, attitudes, perceptions, and values regarding the "nature" and significance of spirituality. This includes the relevance and importance of cognition and spirituality for personal functioning. Experiential/Phenomenological Dimensions of Spirituality taps beliefs about the spiritual, religious, mystical, and transcendental. Existential Well-being involves expressions of spirituality reflected in a sense of meaning and purpose for existence. This includes perceptions of self and understanding and coping with life and the limitations of human existence. Paranormal Beliefs comprises expressions of spirituality concerning beliefs in the paranormal phenomena such as precognition and psychokinesis. This dimension also includes beliefs about witchcraft and spiritualism such as ghosts. Religiousness refers to actual religious practices such as church 
attendance. Some of the statements listed for students to rate on a 5-point scale were, "I believe that going to religious services is important," "I feel a sense of closeness to a higher power,” and “Spirituality is important to who I am as a person.” Cronbach’s alphas for the subscales ranged from .43 to .89 in this sample, and for the entire scale was .75.

\section{Results}

Cognitive orientation towards spirituality and experiential spirituality positively correlated significantly with Observe, a subscale of Mindfulness ( $r=.41, r=.37$, respectively, $\mathrm{p}<.001$ ). Nonjudgment positively correlated with existential well-being ( $r=.28, \mathrm{p}<.05)$. Acting Aware positively correlated with existential well-being $(r=.30, p<.001$, Table 2$)$. The rest of the mindfulness subscales were not significantly correlated with the spiritual expressions subscales (all ps $>0.05$ )

In addition, we explored the relationship of specific paranormal beliefs and the mindfulness subscales. Results showed that experience beyond normal everyday sense of self significantly correlated with Observe, subscale of Mindfulness $(\mathrm{r}=.37, \mathrm{p}<.001)$. Beliefs to predict the future were positively correlated with non-react mindfulness subscale $(r=.30, p<001)$. Beliefs on higher power of force significantly correlated with observe and belief in psychokinesis significantly correlated with describe $(\mathrm{r}=.31, \mathrm{p}<.001)$.

\section{Discussion}

The main finding of this pilot study was that people who usually act as the observer and detach from the events that take place are more likely to be spiritually oriented. Those individuals seem to perceive themselves as spiritual and they are likely to have had a spiritual experience in the past. Non-Judgment or acceptance of everything that comes into perception increases when people feel 
satisfied and happy with themselves. In addition self-awareness seem to be associated with spiritual well being. These findings have important clinical implications given the growth of mindfulness approaches in psychotherapy. Of note, paranormal beliefs seem to correlated with some of mindfulness subscales confirming the idea that people who are mindful are more likely to experience a metaphysical experience such as belief in psychokinesis, “ out of body” experience and predicting the future.

Our preliminary results show that there is a strong connection between spirituality and mindfulness. People that practice mindfulness (the cultivation of a focused, non-judgmental awareness on the present moment), are more inclined to accept new spiritual experiences. Among other mindfulness-based interventions, Mindfulness Based Cognitive Therapy (MBCT) has being widely used in psychotherapy. MBCT was originally developed as a prophylactic intervention for use with people with an established history of relapsing depression. Its effectiveness in reducing the frequency of depression relapse in people who had had three or more depressive episodes has been attributed to a capacity to prevent chronic depressive ruminations (negative thoughts about the past) from maintaining this vulnerability (Teasdale et al, 2000).

However, It must not be taken for granted that all of the clinical results of mindfulness practice are necessarily positive or therapeutic. Attrition during trials of mindfulness-based interventions is not often assessed and the whole question of side effects is under-researched. Possible unintended effects that are known to be exacerbated during intensive training retreats include restlessness, anxiety, depression, guilt and hallucinosis (Albeniz \& Holmes, 2000).

Collectively, Mindfulness is a broad term being used in different psychological approaches. Walking and sitting meditations are common techniques for the mindfulness-based therapeutic approach. Patients are usually encouraged to practice mindfulness in daily life. This continues the 
therapeutic process outside of the limits of the client-therapist session and allows the client the opportunity to observe the mindfulness experience in a non-clinical environment. The clinician and client assess and explore the effects and obstacles encountered during daily life, and use this as a catalyst for behavior and thought modification.

Mindfulness from a psychotherapeutic perspective is not encouraging patients to disconnect from themselves but become aware of all the history and experience that influences them in the present moment. Patients are taught to learn from the past in a nonjudgmental way by cultivating compassion and self-love.

These findings, though preliminary, provide an insight to possible mechanisms that affect individuals suffering from mental health disturbances. Future case studies could potentially provide more information and phenotype who and how patients can benefit from mindfulness.

\section{Conclusions}

Spirituality and mindfulness have both been researched independently. Dearth of information let us void the strong links between the two, however. Spirituality is an experience of mindfulness, while mindfulness is practice to the spiritual. Interconnected and with adequate history to support the links (i.e. roots of mindfulness practice), both concepts concern the spirit, mind and soul, no matter what the definitions of these are. In accordance to religious teachings, spiritual growth coupled with self-awareness and mindfulness are lived experiences that stray away from the existence of the body and focus on the mind and soul. Nonetheless, these experiences are not lived through an inexistent state or object. The body becomes the crucial mean in order to achieve spiritual experiences and a mindful state. Spirituality, linked with mindfulness, cannot be separated from the body. The latter is responsible for being able to have spiritual experiences. 
Spiritual growth is the pattern for believing, whether that is religious belief or not, and mindfulness is a spiritual practice for becoming self-aware of that belief. In a world where the body is admirable for beauty and strength, mindfulness teaches spiritual seekers to accept their physical imperfections and use them as tools for spiritual growth. Apart from sociological and anthropological research, arts and humanities offer a vast number of alternative approaches on the subject, which lead to knowledge by experience, an aspect largely important on this subject. Realization of the potential range and modes of action of mindfulness in therapeutic settings may therefore mean that currently favored methods of investigation need to be complemented by others. These would pay far more detailed and inclusive attention to what happens within and between therapists and patients in terms of awareness during therapeutic sessions.

\section{References}

Albeniz, A. \& Holmes, J. (2000) Meditation: concepts, effects and uses in therapy. International Journal of Psychotherapy, 5, 49-58.

Baer R.A, Smith G.T, Hopkins J., Krietemeyer J., Toney, L. (2006). Using self-report assessment methods to explore facets of mindfulness. Assessment, 13, 27-45.

Bærentsen, K.B., Stødkilde-Jørgensen, H., Sommerlund, B., Hartmann, T., Damsgaard-Madsen, J., Fosnæs, M., \& Green, A.C. (2010). An investigation of brain processes supporting meditation. Cognitive processing, 11(1), 57-84.

Becker, E. (1971). The birth and death of meaning. New York: Free Press.

Berry, D. (2005). Methodological pitfalls in the study of religiosity and spirituality. Western Journal of Nursing Research, 27(5), 628-647. 
Canda, E.R., \& Furman , L.D. (2010). Spiritual diversity in social work practice: The heart of helping (2nd ed.). USA: Oxford University Press.

Davies, D. (2008). The Theology of Death. New York: T\&T Clark.

Day, A. (2011). Believing in Belonging Belief and Social Identity in the Modern World. Oxford \& New York: Oxford University Press.

Day, A. (2010). Propositions and performativity: Relocating belief to the social . Culture and Religion: An Interdisciplinary Journal, 11(1), 9-30.

Desbordes, D., Gard, T., Hoge, E.A., Hölzel, B.K., Kerr, C., Lazar, S.W., Olendzki, A., Vago, D. (2014). Moving Beyond Mindfulness: Defining Equanimity as an Outcome Measure in Meditation and Contemplative Research. Mindfulness, 1-17.

Egan, R., MacLeod, R., Jaye, C., McGee, R., Baxter, J., \& Herbison, P. (2011). What is Spirituality? Evidence from a New Zealand hospice study. Mortality, 16(4), 307-324.

Feifel, H. (1977). New Meanings of Death. New York: McGraw Hill.

Flanagan, K., \& Jupp, P.C. (2009). A Sociology of Spirituality. England: Ashgate.

George, L.K., Larson, D.B., Koenig, H.G., \& McCullough, M.E. (2000). Spirituality and Health: what we know, what we need to know. Journal of Social and Clinical Psychology, 19 (1), 102116.

Harris, J.I., Park, C.L., Currier, J.M., Usset, T.J., \& Voecks, C.D. (2015). Moral injury and psycho-spiritual development: considering the developmental context. Spirituality in Clinical Practice. Advance online publication.

Hervieu-Léger, D. (2000). Religion as a Chain of Memory. USA: Rutgers University Press. 
Hettema, T.L. (2011). Spirituality and Biblical Hermeneutics: The Challenge of Ricoeur’s Philosophy. In J. Verheyden, T.L. Hettema, \& P. VandeCasteele (Eds.). Paul Ricoeur: Poetics and Religion (pp.213-227). Leuven: Peeters.

Hill, P.C., Pargament, K.I., Hood, R.W., McCullough, M.E., Swyers, J.P., Larson, D.B., \& Zinnbauer, B.J. (1999). Conceptualizing Religion and Spirituality: Points of Commonality, Points of Departure. Journal for the Theory of Social Behaviour, 30 (1), 52-77.

James, W. (1961). Varieties of religious experiences. New York: Collier. (Original work published 1902).

Kabat-Zinn, J. (1990). Full catastrophe living: using the wisdom of your body and mind to face stress, pain and illness. New York: Delacorte.

Koenig, H. (2008). Concerns about measuring ‘spirituality’ in research. Journal of Nervous \& Mental Disease, 196(5), 349-355.

MacDonald, D. (2000) Spirituality: measurement and relation to ve factor model of personality. Journal of Personality, 68, 153-197.

McGuire, M.B. (2003). Why Bodies matter: A Sociological Reflection on Spirituality and Materiality. Spiritus: A Journal of Christian Spirituality, 3(1), 1-18.

McSherry, W., \& Ross, L. (2002). Dilemmas of spiritual assessment: considerations for nursing practice. Journal of Advanced Nursing, 38(5), 479-488.

McSherry, W. (2001). Spiritual crisis? Call a nurse. Spirituality in Health Care Contexts, 107117.

Mueller, P. S., Plevak, D. J., \& Rummans, T. A. (2001). Religious involvement, spirituality, and medicine: implications for clinical practice. In Mayo clinic proceedings (Vol. 76, No. 12, pp. 12251235). Elsevier. 
Pentaris, P. (2011). Culture and Death: A Multicultural Perspective. Hawaii Pacific Journal of Social Work Practice, 4(1), 45-84.

Ricoeur, P. (1976). Interpretation Theory: Discourse and the Surplus of Meaning. Texas: Texas Christian University Press.

Rochford, T. (2004). Whare Tapa Wha: A Maori model of a Unified Theory of Health. The Journal of Primary Prevention, 25(1), 41-57.

Schaeffer, F.A. (1972). The new super-spirituality. Michigan: Inter-Varsity Press.

Schalow, F. (2001). Heidegger and the Quest for the Sacred: From Thought to the Sanctuary of Faith (Vol. 44). The Netherlands: Kluwer Academic Publishers.

Sperry, L. (2001). Spirituality in clinical practice: Incorporating the spiritual dimensions in psychotherapy and counseling. Philadelphia, PA: Brunner-Routledge.

Swinton, J., \& Pattison, S. (2010). Moving beyond clarity: Towards a thin, vague, and useful understanding of Spirituality in Nursing Care. Nursing Philosophy, 11(4), 226-237.

Tanyi, R.A. (2002). Towards clarification of the Meaning of Spirituality. Journal of Advanced Nursing, 39(5), 500-509.

Teasdale, J. D., Segal, Z. V., Williams, J. M. G., et al (2000). Prevention of relapse/recurrence in major depression by mindfulness based cognitive therapy. Journal of Consulting and Clinical Psychology, 128, 615-623.

Vachon, M.L. (2008, August). Meaning, spirituality, and wellness in cancer survivors. In Seminars in Oncology Nursing (Vol. 24, No. 3, pp. 218-225). WB Saunders.

Walsh, R. (1992). The search for synthesis: transpersonal psychology and the meeting of east and west, psychology and religion, personal and transpersonal. Journal Humanistic Psychology, 32, 19-45. 
Wallace, B.A., \& Shapiro, S.L. (2006). Mental balance and well-being: building bridges between Buddhism and Western psychology. American Psychology, 61, 690-701

Weaver, A.J., Pargament, K.I., Flannelly, K.J., \& Oppenheimer, J.E. (2006). Trends in the Scientific Study of Religion, Spirituality, and Health: 1965-2000. Journal of Religion \& Health, 45(2), 208-214.

Wilfred, M. (2006). The principal components model: a model for advancing spirituality and spiritual care within nursing and health care practice. Journal of clinical nursing, 15(7), 905-917

Zinnbauer, B.J., Pargament, K.I., \& Scott, A.B. (1999). The Emerging Meanings of Religiousness and Spirituality: Problems and Prospects. Journal of Personality, 67(6), 889-919. 
This is an Accepted Manuscript of an article published by Taylor \& Francis Group in Person-Centered \& Experiential Psychotherapies on 20/06/2016, available online: $\mathrm{http}: / / d x$.doi.org/10.1080/14779757.2016.1180634

Table 1. Sociodemographics

\section{Frequency}

\begin{tabular}{lc} 
\% Female & $65.3 \%$ \\
\% Married & $17.9 \%$ \\
\%Engle & $55.8 \%$ \\
\%College degree & $59.8 \%$ \\
\%Masters degree & $29.3 \%$ \\
18-25 years old & $46.7 \%$ \\
26-34 years old & $10.5 \%$ \\
$35-64$ years old & $55.4 \%$ \\
$55-64$ years old & $30.4 \%$ \\
\hline
\end{tabular}


This is an Accepted Manuscript of an article published by Taylor \& Francis Group in Person-Centered \& Experiential Psychotherapies on 20/06/2016, available online: http://dx.doi.org/10.1080/14779757.2016.1180634

\section{Table 2. Correlations between ESI scales and FFMQ scales}




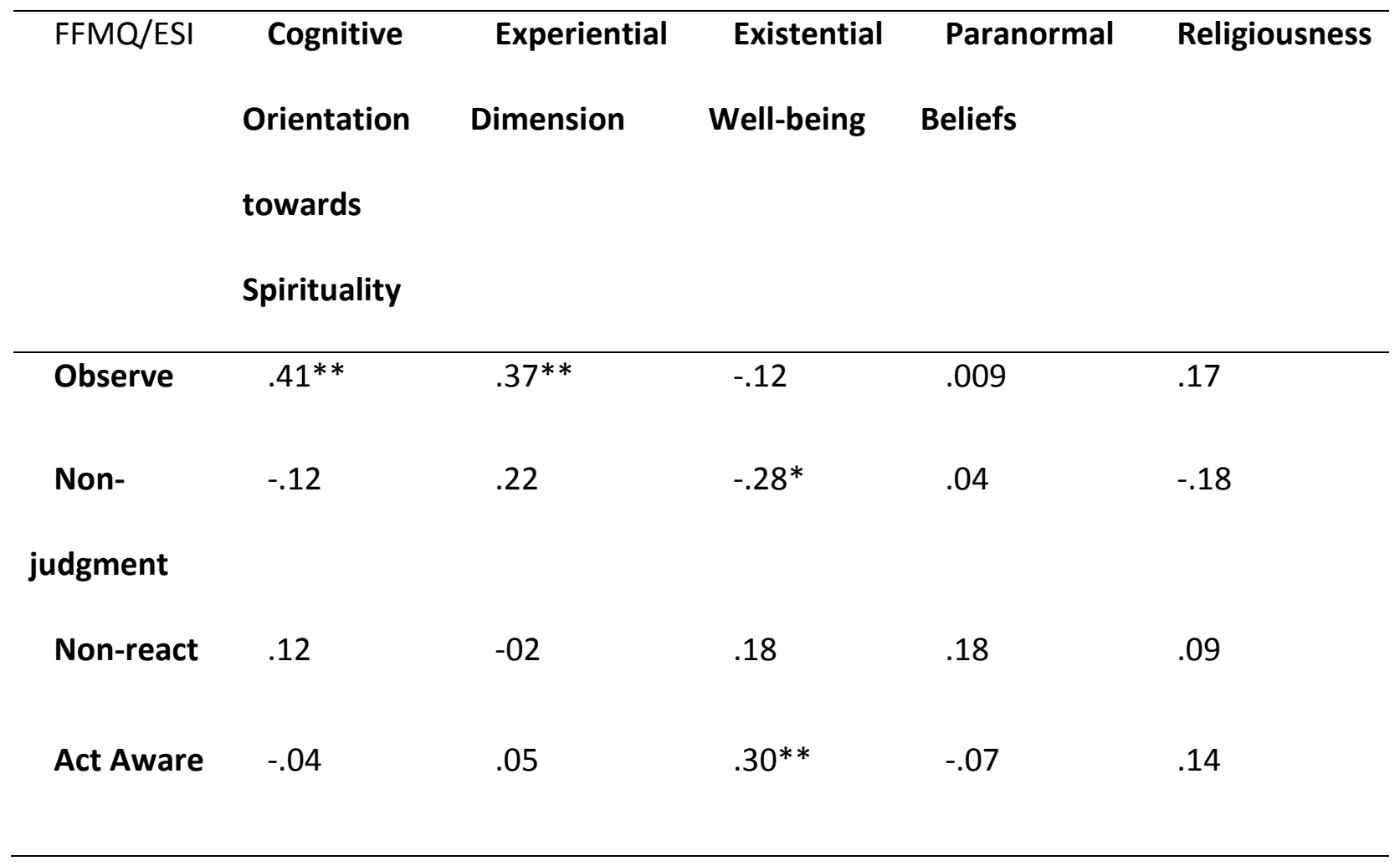

${ }^{*} p<0.05,{ }^{* *} p<.01$ 
This is an Accepted Manuscript of an article published by Taylor \& Francis Group in Person-Centered \& Experiential Psychotherapies on 20/06/2016, available online: http://dx.doi.org/10.1080/14779757.2016.1180634 